\title{
Retinal Hemangioblastoma with Extraocular Extension: Report of Three Cases
}

\author{
Namita Kumari ${ }^{\mathrm{a}}$ Sima Das ${ }^{\mathrm{a}}$ Anirban Bhaduri ${ }^{\mathrm{b}} \quad$ Arpan Gandhi $^{\mathrm{c}}$ \\ ${ }^{a}$ Oculoplasty and Ocular Oncology Services, Dr Shroff's Charity Eye Hospital, New Delhi, India; 'b Oculoplasty and \\ Ocular Oncology Services, Susrut Eye Foundation and Research Institute, Kolkata, India; 'Laboratory Services, \\ Dr Shroff's Charity Eye Hospital, New Delhi, India
}

\section{Established Facts}

- Retinal hemangioblastom is the earliest manifestation of Von Hippel-Lindau disease.

- Retinal hemangioblastoma can progress and can cause vision loss due to exudation and retinal detachment.

\section{Novel Insights}

- Retinal hemangioblastoma can have aggressive behaviour with extraocular extension requiring enucleation in few cases.

- Hemangioblastoma associated with Von Hippel-Lindau as well as isolated retinal hemangioblastoma can have aggressive course.

\section{Keywords}

Hemangioblastoma • Von Hippel-Lindau disease ·

Extraocular extension

\section{Abstract}

Retinal hemangioblastoma $(\mathrm{RH})$ is the earliest and most common clinical manifestation in Von Hippel-Lindau (VHL) disease. $\mathrm{RH}$ can also present in isolation without any evidence of VHL. Clinical course of RH can be stationary or progress to exudation and chronic retinal detachment requiring surgical intervention. We report 3 cases of aggressive $\mathrm{RH}$ with extraocular extension in young males causing painful blind eye requiring enucleation. Two of our cases were bilateral involvement and had systemic manifestations of VHL. The third patient had unilateral involvement with no systemic evidence of VHL. This manifestation of $\mathrm{RH}$ is rarely reported. Two of our patients with VHL had early manifestations of $\mathrm{RH}$ and had undergone multiple cryotherapy sessions as well as retinal detachment surgery for exudative retinal detachment. This differential should be considered in vascular lesion arising from intraocular structures especially in diagnosed patients of VHL. The cases also highlight the aggressive behaviour and long-term progression of $\mathrm{RH}$ in some patients despite early treatment.

(C) 2021 S. Karger AG, Basel karger@karger.com

(c) 2021 S. Karger AG, Basel

www.karger.com/oop

Karger ${ }^{\prime}=$
Sima Das

Dr Shroff's Charity Eye Hospital Daryaganj, New Delhi

5027 Kedar Nath Road

New Delhi 11002 (India)

contactsima@gmail.com 


\section{Introduction}

Von Hippel-Lindau (VHL) disease is an autosomal dominant inherited multisystem disease, arising from germline mutation in VHL gene leading to various manifestations of benign and malignant tumours. It affects the retina and central nervous system, as well as visceral organs, including the kidney, pancreas, liver, and adrenal glands. Retinal hemangioblastomas (RHs) are usually the earliest detected clinical and diagnostic manifestation of VHL disease. The frequency of RH in VHL disease has been reported to range from 25 to $60 \%$ [1]. Mean age at diagnosis of $\mathrm{RH}$ in VHL disease is approximately 25 years, most commonly between 10 and 40 years and extremely rare beyond 60 years [2]. Only $5 \%$ of patients with VHL disease develop RH before 10 years [2]. $\mathrm{RH}$ if not detected and treated early can lead to severe complications like extensive retinal exudation, retinal detachment, retinal neovascularization, and vitreous haemorrhage leading to painful blind eye. We report 3 cases of aggressive $\mathrm{RH}$ with extraocular extension.

\section{Case Report}

Case 1

A 30-year young male presented with progressively increasing painful reddish mass in right eye for past 3 years. He was a known case of VHL disease with blindness in both eyes for past 14 years following multiple retinal surgeries for retinal detachment associated with RH. He was diagnosed as VHL disease based on the presence of bilateral renal cortical cysts and hemangioblastoma of spinal cord both of which were asymptomatic. There was no history of headache, nausea, vomiting, vertigo, abdominal pain, dysuria, haematuria, hearing loss, and aural fullness. Genetic counselling was done. However, screening of family members was not done as he was an adopted child and did not have any biological siblings.

On clinical examination, he had no light perception in both eyes. Right eye showed total ptosis covering an underlying large vascularized mass (shown in Fig. 1a) protruding through superior
Fig. 1. Clinical, imaging, and histopathological findings of case 1. Right upper eyelid total ptosis (a) and reddish-pink fungating intraocular mass in an young adult (b). c CT scan orbits, sagittal section showing heterogeneous intraocular mass with extraocular extension anteriorly along with calcification of the ocular coats. d MRI spine showing spinal hemangioblastoma. e $\mathrm{H}$ and $\mathrm{E}$ stained slides of the histopathology of the enucleated eyeball showing proliferation of capillaries with variable sized, closely packed, thin-walled vessels and large neoplastic stromal cells with pink to clear foamy cytoplasm with fine vacuoles ( $\times 40$ magnification). IHC showed inhibin positivity in foamy cells (f) and CD 34 highlighted the blood vessels ( $\mathbf{g})$. IHC, Immunohistochemistry; CT, computed tomography.
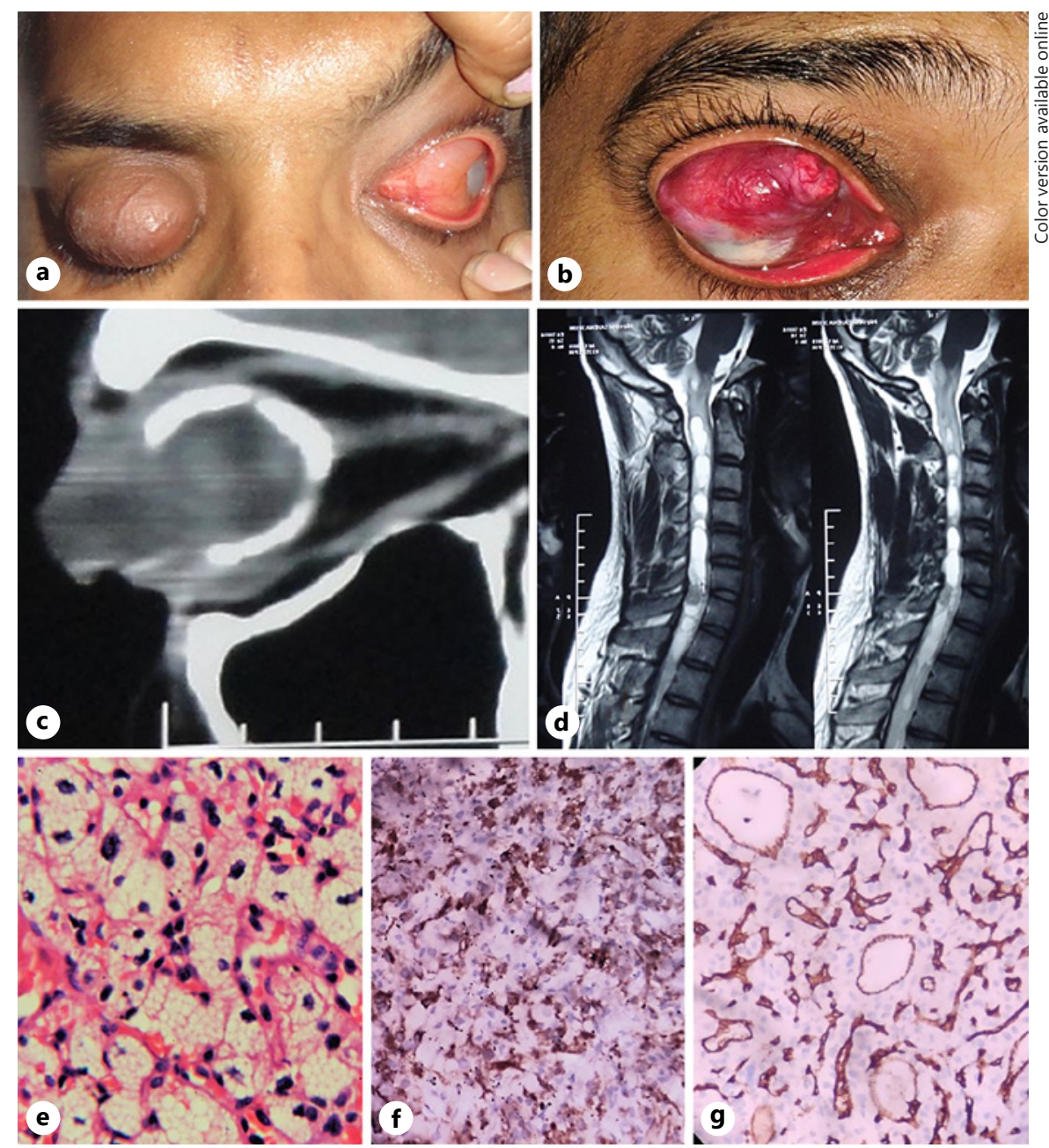
and temporal bulbar conjunctiva with large dilated subconjunctival feeder vessels (shown in Fig. 1b). Opaque cornea was visible inferiorly. Left eye had total corneal opacification with chemosed conjunctiva and subconjunctival silicone oil deposits. Systemic examination revealed hypertension (blood pressure 160/100 mm $\mathrm{Hg}$ ), and he was not on any medication for the same. There was no sensory or motor deficits or any signs of spinal cord compression.

Ultrasound B scan of right eye revealed phthisical eyeball with mushroom-shaped retinal lesion with heterogeneous internal echoes and ocular coats calcification; moderate intensity echoes are seen anteriorly suggesting anterior extension of intraocular lesion. Left eye was silicone filled. Computed tomography (CT) scan orbits revealed grossly distorted bilateral globes with the presence of heterogeneous predominantly hypodense intraocular mass lesion in both eyes with ocular coat calcification (shown in Fig. 1c). Anterior extraocular extension of intraocular lesion was evident in right eye with was no retrobulbar extension in either eye. Ultrasonography of abdomen showed multiple variable size simple cysts in bilateral kidneys and pancreas. MRI of cervical spine showed an elongated solid-cystic mass lesion, isointense on T1w sequence, and hypointense on $\mathrm{T} 2 \mathrm{~W}$ sequence with prominent flow void on the surface of the solid component suggestive of hemangioblastoma with associated syringohydromyelia, extending from C2 to D8 vertebral level (shown in Fig. 1d). MRI brain was normal. Right eye enucleation was done in view of painful blind eye. Histopathological examination revealed an intraocular vascularized lesion covered by conjunctival epithelium that extended through a defect in the superior limbus. The tumour composed of proliferating thinwalled capillaries of variable size, surrounded by stromal cells with pink to clear foamy cytoplasm with fine vacuoles, hyperchromatic nuclei. Numerous mast cells were seen within the tumour mass. The major part of the retina, choroid, and vitreous were replaced by the lesion. No mitotic figures were identified, and optic nerve was unremarkable. Immunohistochemistry (IHC) was positive for CD34, and inhibin was positive in foamy cells. The histopathology and IHC appearance were suggestive of hemangioblastoma (shown in Fig. 1e-g). Patient was advised right eye custom ocular prosthesis post-operatively followed by aponeurotic ptosis correction and was referred to physician for management of hypertension.

\section{Case 2}

A 21-year young male presented with complaints of recurrent redness in the right eye for 5-6 years followed by progressively increasing reddish mass and decreased vision for 5 months. Right eye had no light perception, and left eye BCVA was 20/20. A vascularized subconjunctival mass of size $5 \times 3 \times 3 \mathrm{~mm}$ was noted at inferior limbus and similar smaller nodule at $2^{\prime}$ o clock with dilated episcleral feeder vessels in the right eye (shown in Fig. 2a). Anterior segment examination showed band-shaped keratopathy, corneal scar, hyphema, and no fundal view. Anterior and posterior segment examination of left eye was normal. B scan of right eye showed an ill-defined intraocular mass lesion with heterogeneous internal echoes in inferior quadrant of eye, ocular coats calcification. CT scan orbits showed an ill-defined heterogeneous mass, involving half of vitreous cavity with extraocular extension (shown in Fig. 2b). Ultrasonography of abdomen, MRI brain and spine revealed no abnormality. He underwent right eye enucleation and histopathology showed microscopic features suggestive of hemangioblastoma with extraocular extension anteriorly, and IHC was positive for CD34 and inhibin (shown in Fig. 2c-e). On systemic evaluation, there were no features suggestive of VHL and no significant family history. He was referred for genetic counselling and testing which he refused.

\section{Case 3}

A 37-year-old male was referred with chief complaints of gradually increasing reddish mass in the left eye for last 4 years. He had history of loss of vision in both eyes since childhood following multiple retinal detachment surgeries elsewhere. Fine-needle aspiration cytology done elsewhere from the left eye mass was reported as a malignant spindle cell/poorly differentiated carcinoma and was referred for further management.
Fig. 2. Clinical, imaging, and histopathological findings of case 2. a Vascularized right subconjunctival mass at the inferior limbus with dilated episcleral blood vessels. b CT scan orbits showing ill-defined heterogeneous intraocular mass with areas of calcification within. c Histopathology of the enucleated eyeball ( $\mathrm{H}$ and E staining, $\times 10$ magnification) showing intraocular mass, abundant vascularity with both thick-walled and thin-walled large vessels, and an extensive capillary network and stromal cells are arranged in lobular patterns with abundant foamy cytoplasm and irregular, hyperchromatic nuclei ( $\mathrm{H}$ and $\mathrm{E}$, $\times 40$ magnification). IHC staining with CD34 highlighted the capillary network of tumour vasculature (d) and inhibin was positive in stromal cells (e). IHC, Immunohistochemistry; CT, computed tomography.
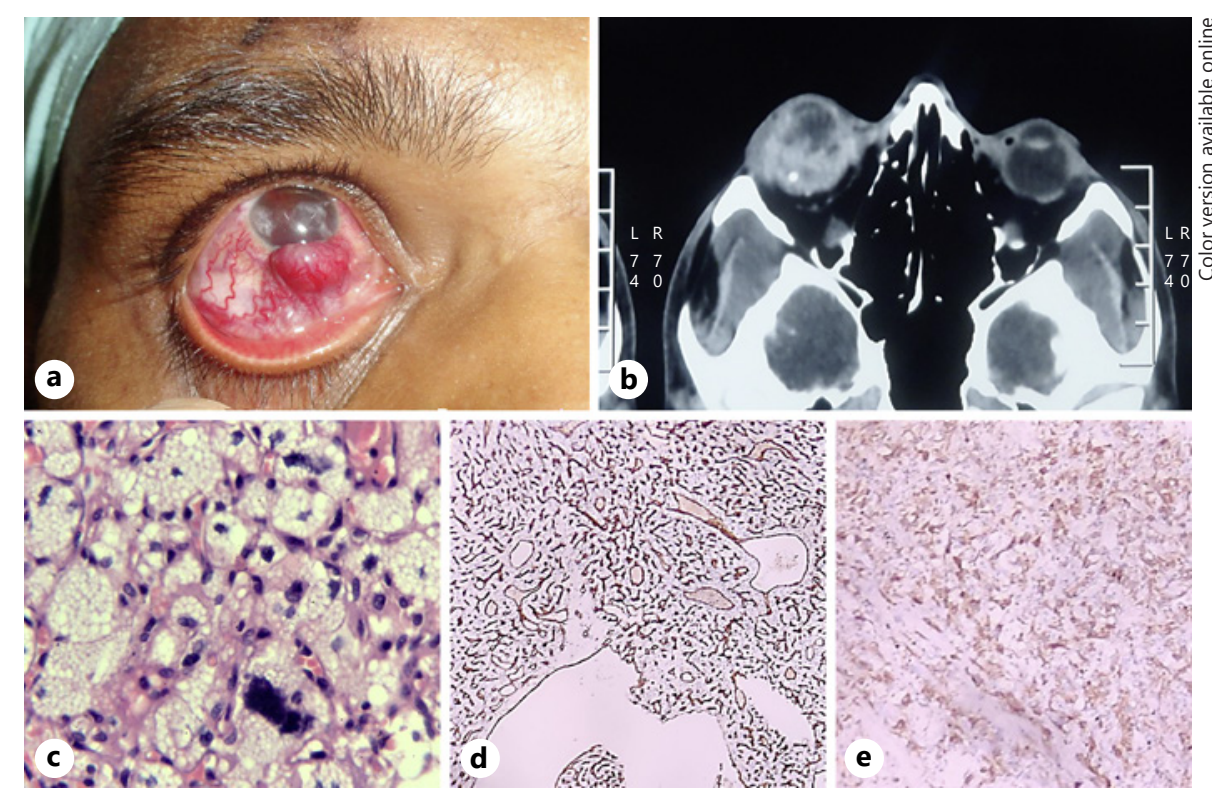
Fig. 3. a Clinical, imaging, and histopathological findings of case 3. Left eye fungating intraocular mass with extraocular extension. b T1W MRI orbits showing left eye intraocular mass with extraocular extension with flow voids within. c MRI brain with contrast showing cystic mass in left cerebellum suggestive of cerebellar hemangioblastoma. d Gross examination of the enucleated eyeball showing a tan-brown intraocular mass with extraocular extension anteriorly. Histopathology $(\times 40 \mathrm{mag}$, $\mathrm{H}$ and $\mathrm{E}$ staining) showing an infiltrative tumour with an anastomotic network of delicate capillary-like blood vessels and conspicuous multivacuolated to foamy appearing stromal cells (e) and CD 34 positivity in vascular walls (f) and inhibin positivity in foamy cells $(\mathbf{g})$.
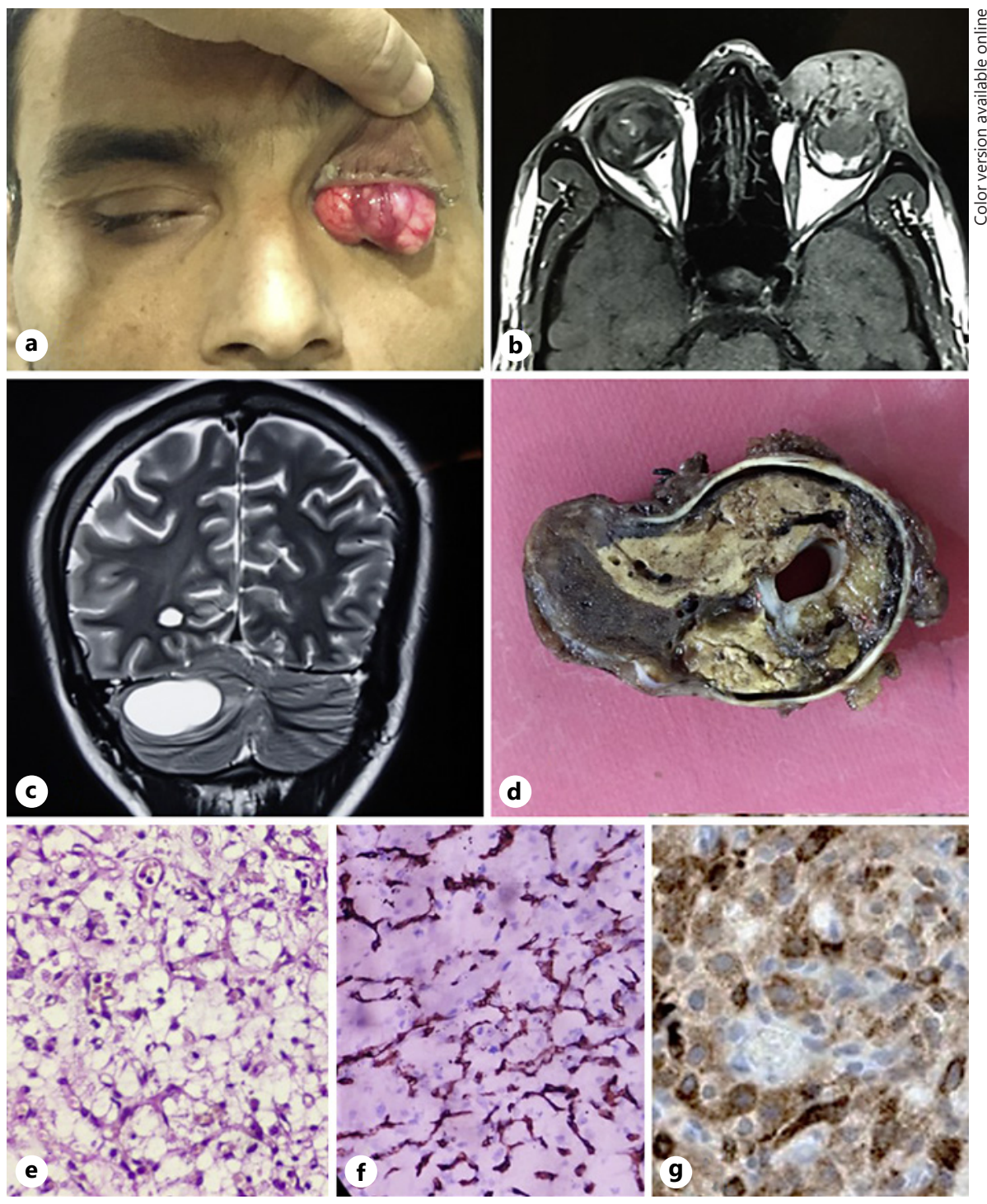

On clinical examination, he denied light perception in both eyes. Right eye showed total corneal opacity with dilated conjunctival vessels superonasally. Left eye had severe ptosis with large reddish lobulated mass visible on lifting the ptotic upper lid (shown in Fig. 3a). MRI orbits showed phthisical eyes with calcification of the coats and an intraocular mass with extraocular extension anteriorly with flow voids within (shown in Fig. 3b). MRI brain with contrast showed a cystic mass in left cerebellum with a small intramural hyperintense nodule suggestive of hemangioblastoma (shown in Fig. 3c). CT scan abdomen showed polycystic pancreas and multiple bilateral simple renal cysts. Enucleation of the left eye was done under general anaesthesia. The mass was seen to arise from the superior limbus, and opaque cornea was identified inferior to it. On histopathology, there was an infiltrative tumour with an anastomotic network of delicate capillary-like blood vessels and conspicuous multivacuolated to foamy inhibin positive vacuolated tumour cells (shown in Fig. 3d-f). A clinico-pathological diagnosis of ocular hemangioblastoma with extraocular extension with VHL was made.
Patient was on regular follow-up thereafter. He developed headache and vomiting a year later for which he underwent craniotomy, and the cerebellar mass was removed. He has completed 1.5 years of follow-up till now and is no systemic symptoms.

\section{Discussion/Conclusion}

VHL disease is one of the most frequently occurring multisystem familial cancer syndromes [3]. It most commonly manifests with vascular tumours in the retina $(\mathrm{RH}) \mathrm{CNS}(\mathrm{CNS}-\mathrm{H})$, and other visceral tumours-like renal cell carcinoma and pheochromocytoma. Renal cysts, sympathetic ganglia paraganglioma, pancreas (pancreatic neuroendocrine tumours or cystadenomas), endolym- 
phatic sac tumour, epididymis (cysts and/or cystadenomas), and broad ligament cysts (and/or cystadenomas) are others features of this disease.

Chew et al. [4] studied 406 patients with VHL disease and found $11.1 \%$ cases with optic nerve involvement but no case with extra scleral extension. Singh et al. [5] in their series of 68 cases of RH did not report any case of scleral extension. Rodrigues et al. [6] first reported a case of 56-year-old female of known VHL disease, having RH which infiltrated the sclera and eye wall and was subsequently managed by enucleation. Recently, Menon et al. [7] has also reported a case of 37-year-old female of VHL disease having fungating bleeding mass with scleral penetration. Patient also had CNS-H and renal cell carcinoma, which were successfully managed by surgery and radiotherapy [7]. Our case series report 3 cases of aggressive RH with extraocular extension and having diverse clinical presentation of VHL disease.

In our series, $\mathrm{RH}$ seems to be earliest clinical presentation of VHL with aggressive course and progression leading to blindness. Two of our patients had bilateral RH and were diagnosed as VHL based on systemic manifestations. However, our second patient had only $\mathrm{RH}$ with extraocular hemangioblastoma with no other systemic involvement. Genetic testing is helpful for establishing diagnosis of VHL disease in such high-risk individuals. Singh et al. [8] found that solitary lesions can occur sporadically in the absence of VHL disease. In patients with solitary RH, younger age at presentation should raise suspicion for VHL [8].

The pathogenesis and the causes for aggressive behaviour of RH in few patients are not understood completely. Wang et al. [9] showed that upregulation of HIF-1a has been detected in $\mathrm{RH}$ with rapid progression, and upregulation of HIF-2a has been found in RH resistant to antiVEGF therapy. Repeated cryotherapy in such aggressive
RH can cause scleral thinning causing extraocular extension of lesion.

VHL disease exhibits age-dependent and incomplete penetrance and variable expression. Regular screening and surveillance are important to diagnose new tumours. Genetic testing is recommended to know the genotype causing aggressive behaviour of tumour.

We report 3 cases of aggressive $\mathrm{RH}$ with extraocular extension requiring enucleation. This differential should be considered in vascular lesion arising from intraocular structures especially in diagnosed patients of VHL. The cases also highlight the aggressive behaviour and longterm progression $\mathrm{RH}$ in some patients despite early treatment.

\section{Statement of Ethics}

This study was conducted ethically in accordance with the World Medical Association Declaration of Helsinki. Subjects have given their written informed consent to publish their case (including publication of images).

\section{Conflict of Interest Statement}

The authors have no conflicts of interest to declare.

\section{Funding Sources}

The authors did not receive any funding.

\section{Author Contributions}

Namita Kumari: acquisition, analysis, and drafting the work. Sima Das: revising the draft and final approval of the version. Anirban Bhaduri: data acquisition and analysis. Arpan Gandhi: data acquisition.

\section{References}

1 Nielsen SM, Rhodes L, Blanco I, Chung WK, Eng C, Maher ER, et al. Von hippel-lindau disease: genetics and role of genetic counseling in a multiple neoplasia syndrome. J Clin Oncol. 2016 Jun;34(18):2172-81.

2 Maher ER, Yates JR, Harries R, Benjamin C, Harris R, Moore AT, et al. Clinical features and natural history of von hippel-lindau disease. Q J Med. 1990 Nov;77(283):1151-63.

3 Aronow ME, Wiley HE, Gaudric A, Krivosic V, Gorin MB, Shields CL, et al. Von hippellindau disease: update on pathogenesis and systemic aspects. Retina. 2019 Dec;39(12): 2243-53.
4 Chew EY. Ocular manifestations of von hippel-lindau disease: clinical and genetic investigations. Trans Am Ophthalmol Soc. 2005 Dec;103:495-511.

5 Singh AD, Nouri M, Shields CL, Shields JA, Perez N. Treatment of retinal capillary hemangioma. Ophthalmology. 2002 Oct;109(10): 1799-806.

6 Rodrigues M, Iliff NT, Eberhart CG, Montaner S, Sodhi A. Scleral penetration of an unusually aggressive case of a retinal hemangioblastoma. Can J Ophthalmol. 2013 Aug;48(4): e67-71.
7 Menon SG, Ramachandran GM, Jacob M, Hegde A, Pai K. Aggressive-fungating retinal hemangioblastoma. J Can Res Ther. : http:// www.cancerjournal.net/preprintarticle. asp?id $=264226$.

8 Singh A, Shields J, Shields C. Solitary retinal capillary hemangioma: hereditary (von hippel-lindau disease) or nonhereditary? Arch Ophthalmol. 2001 Feb;119(2):232-4.

9 Wang Y, Abu-Asab MS, Shen D, Zhuang Z, Chew EY, Chan CC. Upregulation of hypoxia-inducible factors and autophagy in von hippel-lindau-associated retinal hemangioblastoma. Graefes Arch Clin Exp Ophthalmol. 2014 Aug;252(8):1319-27. 